\title{
Microcystic Calcifying Epithelial Odontogenic Tumor
}

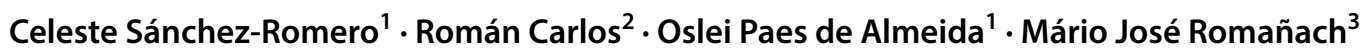

Received: 2 October 2017 / Accepted: 18 November 2017 / Published online: 21 November 2017

(c) Springer Science+Business Media, LLC, part of Springer Nature 2017

\begin{abstract}
Microcystic variant of calcifying epithelial odontogenic tumor is rare. We herein describe an additional well-documented case of microcystic CEOT. The affected patient is a Guatemalan 42-year-old female with an expansile well-defined mixed radiolucent-radiopaque lesion located in the right posterior mandible. The lesion was associated to an unerupted third molar. Histopathologic examination revealed nests and cords of moderately pleomorphic, eosinophilic polyhedral epithelial cells surrounded by a fibromyxoid stroma. The neoplastic cells showed microcystic pattern made of pseudo-glandular spaces with variable diameter. Occasional amyloid deposits and calcified acellular material were observed. Tumor cells were positive for AE1/AE3, CK14, CK19, p63, CD138, and beta-catenin. Conservative surgical resection was performed with an uneventful immediate post-surgical follow-up. After 1 year follow-up there is no evidence of recurrence. Pathologists should be aware of this unusual microcystic presentation of CEOT, which may pose a diagnostic challenge and potential diagnostic dilemma.
\end{abstract}

Keywords Calcifying epithelial odontogenic tumor $\cdot$ Microcystic $\cdot$ Mandible $\cdot$ Immunohistochemistry

\section{Introduction}

Calcifying epithelial odontogenic tumor (CEOT, Pindborg tumor) is an uncommon odontogenic tumor, mainly affecting the mandibular body of individuals during the fifth decade of life with equal sex distribution [1,2]. Most tumors are asymptomatic and slow-growing with a well-defined radiographic appearance, ranging from unilocular to multilocular radiolucencies or mixed radiolucent-radiopaque lesions often showing cortical bone expansion. Approximately 50\% of the reported cases are associated to an unerupted tooth $[3,4]$.

The diagnosis of CEOT is based on the microscopic identification of variable number of nests, sheets or cords

Mário José Romañach

marioromanach@ufrj.br

1 Oral Pathology, Department of Oral Diagnosis, Piracicaba Dental School, University of Campinas, Piracicaba, Brazil

2 Division of Pathology, Centro Clínico de Cabeza y Cuello/Hospital Herrera Llerandi, Guatemala City, Guatemala

3 Oral Pathology, Department of Oral Diagnosis and Pathology, Federal University of Rio de Janeiro School of Dentistry, Av. Carlos Chagas Filho 373, Prédio do CCS Bloco K, $2^{\circ}$ andar Sala 56. Ilha da Cidade Universitária 21.941-902, Rio de Janeiro, Brazil of polyhedral epithelial cells with ample eosinophilic cytoplasm surrounded by a well-defined cellular membrane [1-4]. Prominent intercellular bridges and nuclear pleomorphism are common findings. Often, neoplastic epithelial cells secrete odontogenic amyloid protein, which tends to calcify forming characteristic concentric structures morphologically resembling Liesegang rings [1-4]. Complete surgical removal including a narrow rim of surrounding bone is the current recommended treatment for CEOT. Long-term follow-up is recommended considering a recurrence rate of up to $15 \%$ [5].

Since the first report 70 years ago, different histomorphological features of CEOT have been described, including peripheral location $(6 \%)$, clear cell variant, variable quantity of Langerhans cells and calcified material, multifocal presentation and an exceedingly rare malignant variant [5-9]. Additionally, CEOT-like areas have been described in adenomatoid odontogenic tumors (AOT), but they are considered part of the spectrum of AOT, with its same biological potential. Often, CEOT-like epithelial nests may be observed in dental follicles, and its significance even not completely understood, are not considered early stages of CEOT [1, 2, 7]. Although most cases are solid tumors, rare predominantly cystic CEOT cases have been reported (Table 1) [10-14]. We herein present a well-documented 


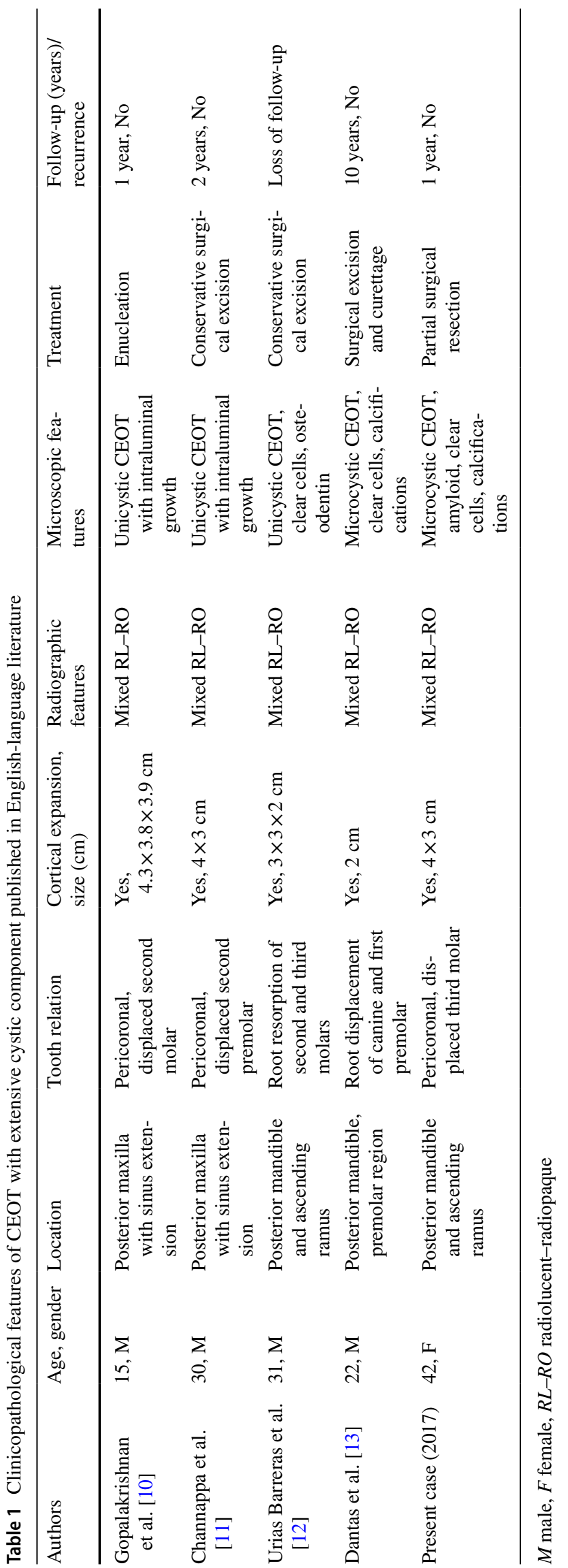

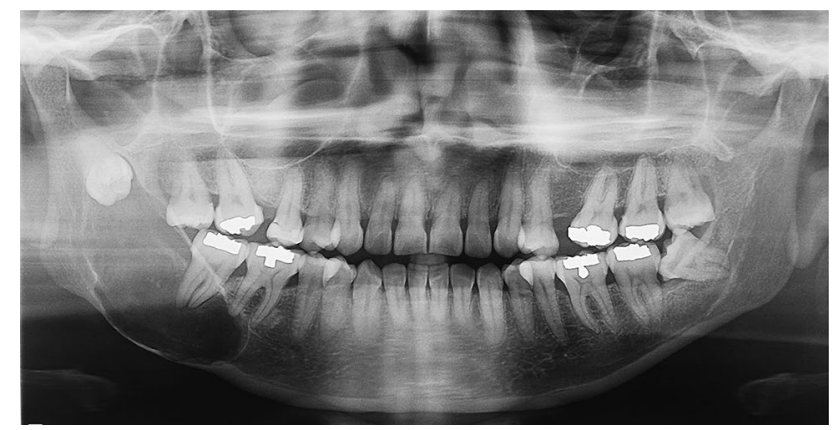

Fig. 1 An expansive and well-defined mixed radiolucent-radiopaque lesion located in the ascending ramus of the mandible, in relation to an unerupted third molar. Cortical bone expansion and thinning of the inferior cortical bone is evident

case of microcystic CEOT located in the posterior mandible of a 42-year-old female.

\section{Case Report}

A 42-year-old female presented with an intra-osseous asymptomatic lesion in the right posterior mandible discovered 3 months prior to consultation. Patient had an otherwise non-contributory past medical history. Cortical bone expansion was evident during intra-oral examination, covered by intact mucosa. Panoramic X-ray revealed a well-defined and corticated mixed radiolucent-radiopaque lesion, measuring approximately $4 \times 3 \mathrm{~cm}$. The lesion was related to an unerupted third molar, which was posteriorly displaced close to the coronoid process. The mandibular nerve canal was basally displaced. Adjacent molars showed intact roots without resorption (Fig. 1). An incisional intraoral biopsy was performed under local anesthesia. Microscopic examination revealed multiple nests and cords of eosinophilic polyhedral epithelial cells supported by fibromyxoid stroma, exhibiting multiple microcystic spaces of varying size, with amyloid-like deposits, which were positive for Congo red and showed green birefringence under polarized light. Prominent intercellular bridges were clearly evident. Nuclei showed mild to moderate pleomorphism, mainly those surrounding the pseudo-ductal spaces. This unusual pattern may mimic metastatic adenocarcinoma or a primary carcinoma of the jaws. Occasional clear cells and minimal concentric calcifications were found (Fig. 2). More than $80 \%$ of the tumor cells showed immunohistochemical positivity for AE1/ AE3 (dilution 1:500, clone AE1/AE3, Dako, Carpinteria, CA, USA), CK14 (dilution 1:200, clone LL002, Novocastra, Newcastle upon Tyne, UK), and CK19 (dilution 1:200, clone RCK108, Dako) in a cytoplasmic pattern, for p63 (dilution 1:300, clone 4A4, Dako) in a nuclear pattern, 

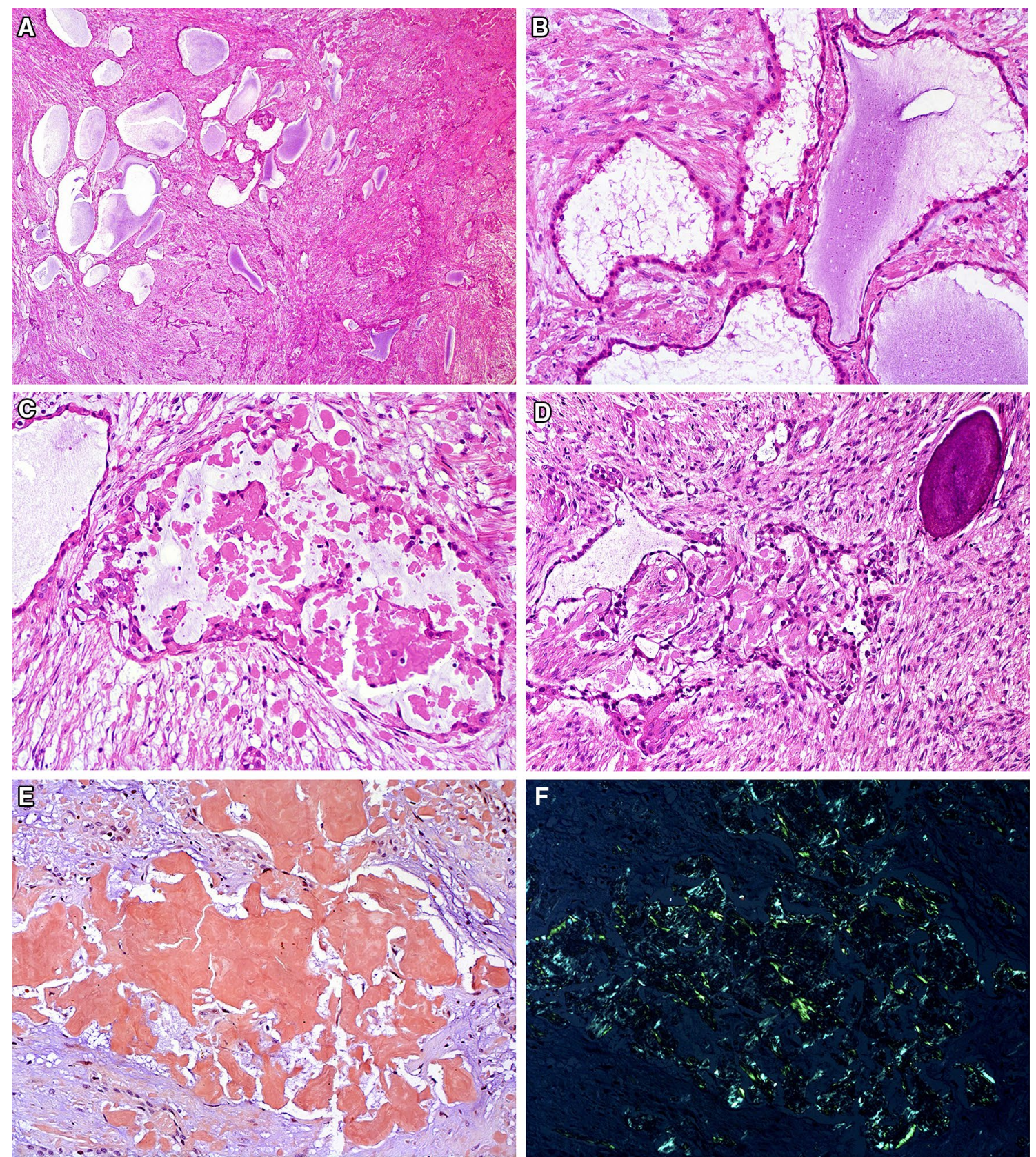

Fig. 2 a, b Microcystic compartments of varying size resembling pseudo-glandular structures in a fibromyxoid stroma. c Extracellular amyloid deposits within a pseudo-ductal structure were $\mathbf{d}$ intermingled with typical cords of polyhedral eosinophilic epithelial cells and

close to calcified material. e, f Congo red positive for amyloid material, which showed green birefringence under polarized light (HE, a $\times 25, \mathbf{b}-\mathbf{f} \times 200$ )

and for CD138 (dilution 1:100, clone MI15, Dako), and beta-catenin (dilution 1:200, clone 17C2, Novocastra) in a membrane pattern. Tumor cells were negative for CK7 (dilution 1:300, clone OV-TL 12/30, Dako) (Fig. 3). The Ki-67 (dilution 1:100, clone MIB-1, Dako) labelling index was less than $1 \%$ after evaluation of 1000 cells per five

high-power fields. The histomorphological and radiographical findings supported a final diagnosis of CEOT, microcystic variant. The patient was treated by conservative surgical resection and microscopic examination of the resected specimen confirmed the initial diagnosis. After 1 year follow-up there is no evidence of recurrence. 

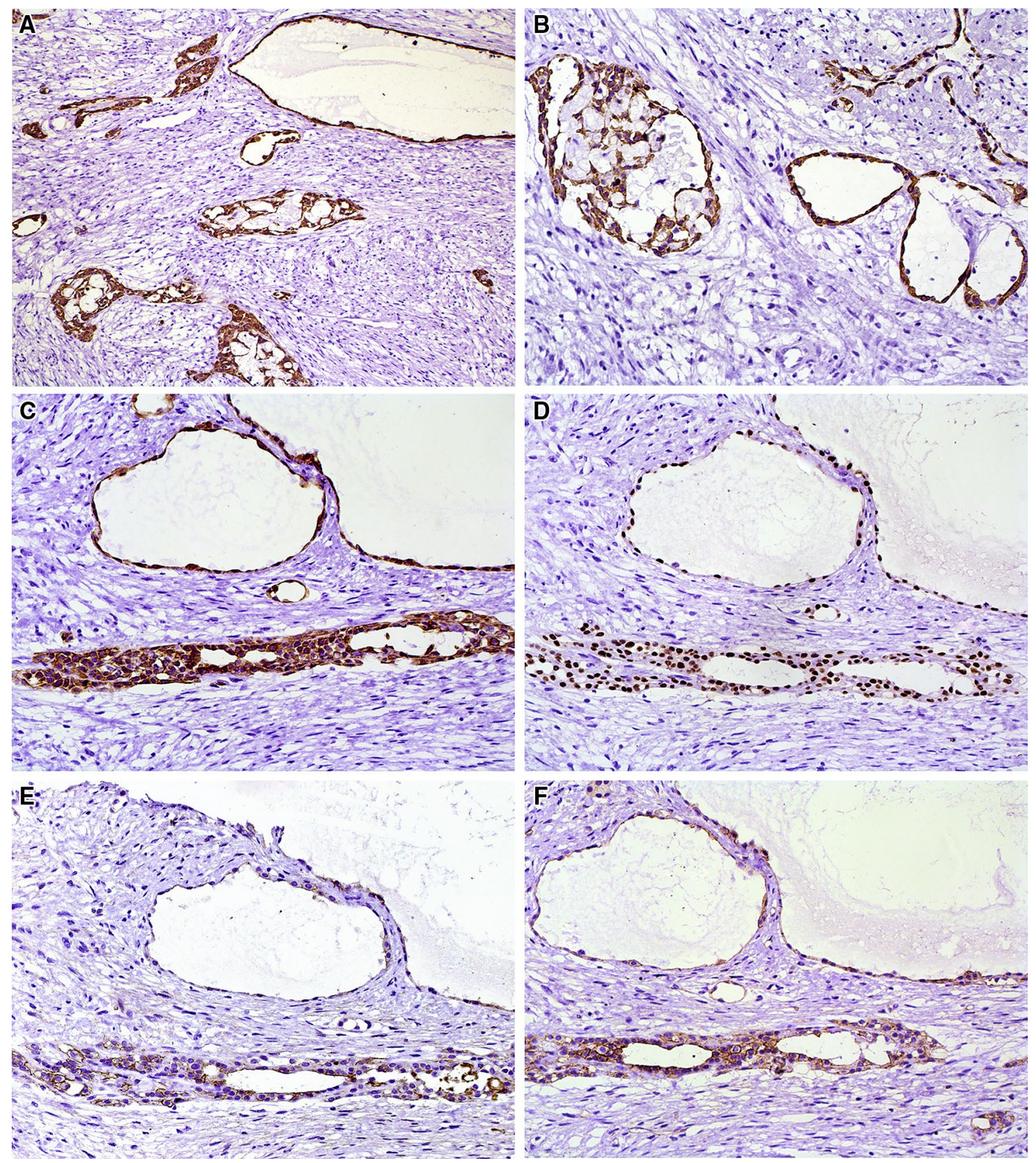

Fig. 3 Tumor cells were positive for a AE1/AE3, b CK14, c CK19, d p63, e CD138, and $\mathbf{f}$ beta-catenin (IHC, ×200)

\section{Discussion}

Virtually any epithelial lesion has the potential to develop cystic or pseudo-cystic spaces, particularly, epithelial odontogenic tumors such as ameloblastoma and adenomatoid odontogenic tumor [10]. On the other hand, cystic lesions e.g. calcifying odontogenic cyst, odontogenic keratocyst or glandular odontogenic cyst, often have aggressive biological behavior, comparable to a solid neoplasm. The difference between an odontogenic cyst and an odontogenic neoplasm may be subtle and difficult. Therefore, close correlation between clinical, radiographic, and gross features are required for proper diagnosis, treatment, and follow-up.

In 2006, Gopalakrishnan et al. reported the first case of cystic variant of CEOT in a 15-year-old male with an expansive mixed lesion of the posterior maxilla [10]. Since then, only three additional CEOT cases showing considerable cystic or microcystic pattern have been reported [11-13]. All patients were males with a median age of 24.5 years (ranging from 15 to 31 ). Radiographically, 
all CEOT with cystic spaces, showed a mixed radiolucent-radiopaque appearance. Two cases were located in the posterior maxilla, related to an unerupted displaced tooth, and showing maxillary sinus extension $[10,11]$, which is also a common finding in conventional maxillary CEOTs [5]. Other two cases were located in the toothbearing regions of the body of the mandible with root displacement or resorption, one of them extending posteriorly into the ramus $[12,13]$. Interestingly, all cystic CEOT cases showed gross expansion of cortical bone, average size was $3.3 \mathrm{~cm}$ in the greatest diameter. To the best of our knowledge, our case is the second microcystic variant of CEOT reported [13].

Microscopically, most CEOT can be readily diagnosed on routine H-E sections, which in some cases required Congo red stain, in order to demonstrate amyloid deposits. Immunohistochemistry is mainly performed for academic reasons and to better understand the pathogenesis of these tumors. At present, the different histologic variants described so far, do not have any impact on prognosis or treatment. However, we consider that is important to describe them, for the benefit of morphologic diagnosis and to prevent potential confusion with other epithelial tumors affecting gnathic bones. For this specific case report, our interest is to highlight this unusual microcystic variant, which may be confused mainly with metastatic adenocarcinoma or other benign or malignant odontogenic tumors. Variable amounts of polyhedral eosinophilic epithelial cells, clear cells, amyloid material and calcifications can be observed in CEOT [10-13]. These features may help to confirm the diagnosis of microcystic CEOT.

As previously mentioned, the diagnostic value of immunohistochemistry in odontogenic lesions is limited [14]. Our results confirmed previously reported findings showing positivity for AE1/AE3, CK14 and CK19. At present, only six reported cases of CEOT were positive for p63 protein $[6,15,16]$, which was also expressed in our case. Similarly, the positivity for syndecan-1 (CD138) and betacatenin in CEOT were observed in only few studies [6, 7], which were both strongly positive in the present case. Therefore, the immunohistochemical profile seems to be similar in all variants of CEOT.

Additional cases of microcystic and other variants of CEOT may contribute to better understand the clinical, histological and biological spectrum of this tumor.

Author Contributions CS-R contributed to the clinical and microscopic analyses of the case studied and review of the literature. RC, OPA and MJR contributed to the study design, final data analyses and manuscript writing and editing. All authors gave final approval and agreed to be accountable for all aspects of the work.

Funding There was no funding for this study.

\section{Compliance with Ethical Standards}

Conflict of interest All of authors have indicated they have no potential conflicts of interest and no financial relationships relevant to this article to disclose.

Ethical Approval This article does not contain any studies with animals performed by any of the authors. All procedures performed in this study involving human participants were in accordance with the ethical standards of the institutional and/or national research committee and with the 1964 Helsinki declaration and its later amendments or comparable ethical standards.

Informed Consent Informed consent was obtained from all individual participants included in the study.

\section{References}

1. Wright JM, Devilliers P. Calcifying epithelial odontogenic tumour. In: El-Naggar AK, Chan JKC, Grandis JR, Takata T, Slootweg PJ, editors. World Health Organization classification of head and neck tumours. Lyon: IARC; 2017. pp. 220-21.

2. Philipsen HP, Reichart PA. Calcifying epithelial odontogenic tumour: biological profile based on 181 cases from the literature. Oral Oncol. 2000;36(1):17-26.

3. Goode RK. Calcifying epithelial odontogenic tumor. Oral Maxillofac Surg Clin North Am. 2004;16(3):323-31.

4. Franklin CD, Pindborg JJ. The calcifying epithelial odontogenic tumor. A review and analysis of 113 cases. Oral Surg Oral Med Oral Pathol. 1976;42(6):753-65.

5. Chrcanovic BR, Gomez RS. Calcifying epithelial odontogenic tumor: an updated analysis of 339 cases reported in the literature. J Craniomaxillofac Surg. 2017;45(8):1117-23.

6. Turatti E, Brasil J, de Andrade BA, Romañach MJ, de Almeida OP. Clear cell variant of calcifying epithelial odontogenic tumor: case report with immunohistochemical findings. J Clin Exp Dent. 2015;7(1):e163-6.

7. Azevedo RS, Mosqueda-Taylor A, Carlos R, Cabral MG, Romañach MJ, de Almeida OP, Pires FR. Calcifying epithelial odontogenic tumor (CEOT): a clinicopathologic and immunohistochemical study and comparison with dental follicles containing CEOT-like areas. Oral Surg Oral Med Oral Pathol Oral Radiol. 2013;116(6):759-68.

8. Afrogheh A, Schneider J, Mohamed N, Hille J. Calcifying epithelial odontogenic tumour with clear langerhans cells: a novel variant, report of a case and review of the literature. Head Neck Pathol. 2014;8(2):214-9.

9. Sedghizadeh PP, Wong D, Shuler CF, Linz V, Kalmar JR, Allen CM. Multifocal calcifying epithelial odontogenic tumor. Oral Surg Oral Med Oral Pathol Oral Radiol Endod. 2007;104(2):e30-4.

10. Gopalakrishnan R, Simonton S, Rohrer MD, Koutlas IG. Cystic variant of calcifying epithelial odontogenic tumor. Oral Surg Oral Med Oral Pathol Oral Radiol Endod. 2006;102(6):773-7.

11. Channappa NK, Krishnapillai R, Rao JB. Cystic variant of calcifying epitelial odontogenic tumor. J Investig Clin Dent. 2012;3(2):152-6.

12. Urias Barreras CM, Quezada Rivera D, Koutlas IG, Gaitán Cepeda LA. Clear cell cystic variant of calcifying epithelial odontogenic tumor. Head Neck Pathol. 2014;8(2):229-33.

13. Dantas RC, Ramos-Perez FM, Perez DE, Durighetto AF Jr, Vargas PA. Cystic variant of calcifying epithelial odontogenic tumor. J Craniofac Surg. 2015;26(5):1722-3. 
14. Hunter KD, Speight PM. The diagnostic usefulness of immunohistochemistry for odontogenic lesions. Head Neck Pathol. 2014;8(4):392-9.

15. Gratzinger D, Salama ME, Poh CF, Rouse RV. Ameloblastoma, calcifying epithelial odontogenic tumor, and glandular odontogenic cyst show a distinctive immunophenotype with some myoepithelial antigen expression. J Oral Pathol Med. 2008;37(3):177-84.

16. Friedrich RE, Zustin J. Calcifying epithelial odontogenic tumour of the maxilla: a case report with respect to immunohistochemical findings. In Vivo. 2011;25(2):259-64. 